\title{
ON REGULARITY CONDITIONS FOR RANDOM FIELDS
}

\author{
RICHARD C. BRADLEY
}

(Communicated by Lawrence Gray)

\begin{abstract}
Indexed by the integer lattice of dimension at least two, there exists a nondegenerate strictly stationary random field which is one-dependent with respect to "lattice-halfspaces" but which is also measurable with respect to its own tail sigma-field.
\end{abstract}

\section{INTRODUCTION}

Suppose $X:=\left(X_{k}, k \in \mathbf{Z}^{d}\right)$ is a random field indexed by the $d$-dimensional integer lattice, on a probability space $(\Omega, \mathscr{F}, P)$. For any nonempty set $S \subset$ $\mathbf{Z}^{d}$, let $\sigma\left(X_{k}, k \in S\right)$ denote the $\sigma$-field of events generated by $\left(X_{k}, k \in S\right)$.

For every $i \in\{1,2, \ldots, d\}$ and every integer $m$, define the sets

$$
G_{i, m}:=\left\{k:=\left(k_{1}, \ldots, k_{d}\right) \in \mathbf{Z}^{d}: k_{i} \leq m\right\}
$$

and

$$
H_{i, m}:=\left\{k:=\left(k_{1}, \ldots, k_{d}\right) \in \mathbf{Z}^{d}: k_{i} \geq m\right\} .
$$

The random field $X$ will be said to be "lattice-halfspace one-dependent" if the following holds: For every $i \in\{1, \ldots, d\}$ and every integer $m$, the $\sigma$-fields $\sigma\left(X_{k}, k \in G_{i, m}\right)$ and $\sigma\left(X_{k}, k \in H_{i, m+2}\right)$ are independent.

For the random field $X$, the "tail $\sigma$-field" is

$$
\mathscr{T}:=\bigcap_{S} \sigma\left(X_{k}, k \in \mathbf{Z}^{d}-S\right),
$$

where the intersection is taken over all finite subsets $S \subset \mathbf{Z}^{d}$. (Note that for a random sequence, i.e., the case $d=1$, this $\sigma$-field $\mathscr{T}$ is the "double" tail $\sigma$-field.) The random field $X$ will be said to be "tail deterministic" if all of its random variables $X_{k}, k \in \mathbf{Z}^{d}$, are measurable with respect to $\mathscr{T}$.

Theorem 1. Suppose $d \geq 2$ is an integer. Then there exists a nondegenerate strictly stationary random field $X:=\left(X_{k}, k \in \mathbf{Z}^{d}\right)$ which is both "latticehalfspace one-dependent" and "tail deterministic".

That is our main result. It is motivated by various conditions of weak dependence (e.g., mixing conditions) that have been used in the study of limit

Received by the editors September 12, 1992.

1991 Mathematics Subject Classification. Primary 60G60; Secondary 60G10.

Key words and phrases. One-dependent, deterministic, strictly stationary random field. 
theory for random fields (see, e.g., [3, 5, 6, 9, 10, 11, 13, 16, 17, 19]). In some papers, the amount of "dependence" (according to some criterion) between the $\sigma$-fields $\sigma\left(X_{k}, k \in G_{i, m}\right)$ and $\sigma\left(X_{k}, k \in H_{i, n}\right)$ is assumed to approach zero as $n-m \rightarrow \infty$. In some other papers, the amount of "dependence" between $\sigma\left(X_{k}, k \in G\right)$ and $\sigma\left(X_{k}, k \in H\right)$, where the sets $G$ and $H$ are essentially "arbitrary", is assumed to approach zero as the "minimum distance" between $G$ and $H$ becomes large. This latter type of condition is the more stringent one. The purpose of Theorem 1 is to provide an (in some sense) extreme "separation" of these two types of conditions.

Bulinskii [6, Theorem 2.1, p. 13] earlier also constructed a (not strictly stationary) random field $X:=\left(X_{k}, k \in \mathbf{Z}^{d}\right)$ to "separate" these two types of conditions. Without going into technical details, let us describe the two main properties of his construction.

(1) With respect to "lattice-halfspaces" $\left(G_{i, m}\right.$ and $\left.H_{i, n}\right)$, his random field satisfies the (Rosenblatt) "strong mixing" condition, with any arbitrary prescribed mixing rate except for (lattice-halfspace) $m$-dependence.

(2) With respect to general index sets $G$ and $H$, his random field fails to satisfy "strong mixing". The random field $X$ in our Theorem 1 complements property (1) of Bulinskii's construction and strengthens property (2).

Various other connections (or lack of them) between various conditions of weak dependence on random fields have been examined, e.g., in $[2,4,5,6,8$, $18,19]$.

Of course, Theorem 1 fails for random sequences, i.e., the case $d=1$. In $[1,7,12,14,15]$ nondegenerate strictly stationary random sequences are constructed which have trivial "past" and "future" tail $\sigma$-fields or even satisfy some strong mixing condition but which have a nontrivial "double" tail $\sigma$-field or are even "(double) tail deterministic".

Theorem 1 is proved in $\S 3$. That proof will be based on preliminary work done in $\S 2$. Our construction will mimic the one in $[14$, p. 155 , lines $5-10$, and p. 156, lines 4-12].

For typographical convenience, products like $\prod_{k \in S} a_{k}$ will sometimes be written $\prod\left[a_{k}: k \in S\right]$, and a similar notation will be used for sums.

\section{Preliminaries}

This section is devoted to a proof of

Proposition 1. Suppose $d \geq 2$ is an integer. Then there exists a (nonstationary) random field $X:=\left(X_{k}, k \in \mathbf{Z}^{d}\right)$ which is both "lattice-halfspace one-dependent" and "tail deterministic" such that each $X_{k}$ takes only the values -1 and +1 , with $P\left(X_{k}=-1\right)=P\left(X_{k}=1\right)=\frac{1}{2}$.

In $\S 3$ this random field $X$ will be "converted" into a stationary one. Our (nonstationary) construction for Proposition 1 will resemble the one in $[14, p$. 155, lines 5-10], which was attributed there to David Freedman.

Proof of Proposition 1. For every $k:=\left(k_{1}, \ldots, k_{d}\right) \in \mathbf{Z}^{d}$, define the "sup norm" $\|k\|:=\max _{1 \leq i \leq d}\left|k_{i}\right|$.

For each $n=0,1,2, \ldots$, define the set $S_{n}:=\left\{k \in \mathbf{Z}^{d}:\|k\|=n\right\}$.

Let $\mathbf{N}$ denote $\{1,2,3, \ldots\}$. Let $\phi: \mathbf{N} \rightarrow \mathbf{Z}^{d}$ be a one-to-one correspondence such that $\|\phi(n)\|$ is nondecreasing as $n$ increases. Obviously this 
forces $\phi(1)=(0,0, \ldots, 0), \phi(n) \in S_{1}$ for $2 \leq n \leq 3^{d}, \phi(n) \in S_{2}$ for $3^{d}+1 \leq n \leq 5^{d}$, and so on. Clearly one has

$$
\|\phi(n)\|<n \quad \forall n \in \mathbf{N} .
$$

Define the set $T_{0}:=S_{0}=\{(0, \ldots, 0)\}$, and for each $n \in \mathbf{N}$ define the set $T_{n}:=S_{n}-\{(n, 0, \ldots, 0)\}$. Then $T_{0} \cup T_{1} \cup T_{2} \cup \cdots=\mathbf{Z}^{d}-\{(n, 0, \ldots, 0)$ : $n \in \mathbf{N}\}$. Let $\left(X_{k}, k \in T_{0} \cup T_{1} \cup T_{2} \cup \cdots\right)$ be i.i.d. r.v.'s taking only the values -1 and +1 , with $P\left(X_{k}=-1\right)=P\left(X_{k}=1\right)=\frac{1}{2}$.

To complete the definition of the random field $X$, we shall define the r.v.'s $X_{(n, 0, \ldots, 0)}, n \in \mathbf{N}$, by the formula

$$
X_{(n, 0, \ldots, 0)}:=X_{\phi(n)} \cdot \prod\left[X_{k}: k \in T_{n}\right] .
$$

Since $\phi(1)=(0, \ldots, 0), X_{(1,0, \ldots, 0)}$ is well defined. Now suppose $n \geq 2$. Then $m:=\|\phi(n)\|<n$ by $(2.1)$. If $\phi(n) \in T_{m}$, then $X_{(n, 0, \ldots, 0)}$ is well defined. If instead $\phi(n)=(m, 0, \ldots, 0)$ and $X_{(m, 0, \ldots, 0)}$ is already defined, then $X_{(n, 0, \ldots, 0)}$ is well defined. Thus (2.2) can be used recursively (where necessary) to define $X_{(n, 0, \ldots, 0)}, n \in \mathbf{N}$. This completes the definition of the random field $X$.

Note that for all $k \in \mathbf{Z}^{d}$ we have that $X_{k}$ takes only the values -1 and +1 . (For $k=(n, 0, \ldots, 0), n \in \mathbf{N}$, again use (2.2) recursively where necessary.) This fact will be used freely in what follows. In particular, it implies that $X_{k}=1 / X_{k}$ for each $k$, and by (2.2)

$$
X_{\phi(n)}=\prod\left[X_{k}: k \in S_{n}\right] \quad \forall n \in \mathbf{N} \text {. }
$$

Lemma 1. For each $m=0,1,2, \ldots$,

$$
\sigma\left(X_{k}, k \in S_{m}\right) \subset \sigma\left(X_{k}, k \in S_{m+1} \cup S_{m+2} \cup S_{m+3} \cup \cdots\right) \text {. }
$$

Proof. Suppose $m \geq 0$ and $j \in S_{m}$. Let $n \in \mathbf{N}$ be the integer such that $\phi(n)=j$. By (2.1), $m=\|j\|<n$, and, by (2.3), $X_{j}$ is $\sigma\left(X_{k}, k \in S_{n}\right)$ measurable. Thus $X_{j}$ is $\sigma\left(X_{k}, k \in S_{m+1} \cup S_{m+2} \cup \cdots\right)$-measurable. Since $j \in S_{m}$ was arbitrary, Lemma 1 follows.

As a consequence of Lemma 1 , we have that the random field $X$ is "tail deterministic", which is one of the required properties in Proposition 1.

Lemma 2. For each $n \in \mathbf{N}$, the r.v. $X_{(n, 0, \ldots, 0)}$ is measurable with respect to $\sigma\left(X_{k}, k \in T_{0} \cup T_{1} \cup \cdots \cup T_{n}\right)$ and $P\left(X_{(n, 0, \ldots, 0)}=-1\right)=P\left(X_{(n, 0, \ldots, 0)}=1\right)=\frac{1}{2}$. Proof. Suppose $n \in \mathbf{N}$. By (2.1) and $(2.2), X_{(n, 0, \ldots, 0)}$ is the product of the $X_{k}$ 's, $k \in T_{n}$, and $X_{j}$ for some $j$ such that $m:=\|j\|<n$. It suffices to establish the fact that $X_{j}$ is $\sigma\left(X_{k}, k \in T_{0} \cup \cdots \cup T_{n}\right)$-measurable and that the $X_{k}$ 's, $k \in T_{n}$, and $X_{j}$ are i.i.d., taking the values -1 and +1 with probability $\frac{1}{2}$ each. We already have the fact if $j \in T_{m}$. If instead $m \geq 1$ and $j=$ $(m, 0, \ldots, 0)$, then in this case too we still have the fact, if we first assume that the conclusion of the lemma is already established for the integer $m$. Thus Lemma 2 can be proved by using induction.

To complete the proof of Proposition 1, all that remains is to prove that the random field $X$ is "lattice-halfspace one-dependent".

First note that by Lemma 2 one has

$$
\sigma\left(X_{k}, k \in S_{0} \cup \cdots \cup S_{n}\right)=\sigma\left(X_{k}, k \in T_{0} \cup \cdots \cup T_{n}\right) \quad \forall n=0,1,2, \ldots
$$


Lemma 3. Suppose $n \in \mathbf{N}$. Suppose $U$ is a nonempty subset of $S_{n}$ such that (to avoid trivialities) $U \neq S_{n}$. Then the $\sigma$-fields $\sigma\left(X_{k}\right), k \in S_{n}-U$, and $\sigma\left(X_{k}, k \in S_{0} \cup S_{1} \cup \cdots \cup S_{n-1}\right)$ are independent.

Proof. It suffices to prove this in the case where $U$ has just one element, which we shall cali $h$.

Case I. $h=(n, 0, \ldots, 0)$. Then by (2.4) Lemma 3 asserts that the $\sigma$-fields $\sigma\left(X_{k}\right), k \in T_{n}$, and $\sigma\left(X_{k}, k \in T_{0} \cup \cdots \cup T_{n-1}\right)$ are independent. But we already have this trivially.

Case II. $h \neq(n, 0, \ldots, 0)$. First note that by $(2.4)$ we already have that the $\sigma$-fields $\sigma\left(X_{k}\right), k \in T_{n}-\{h\}$, and $\sigma\left(X_{k}, k \in S_{0} \cup \cdots \cup S_{n-1}\right)$ are independent. Defining the set $Q:=S_{0} \cup \cdots \cup S_{n-1} \cup\left(T_{n}-\{h\}\right)$ and the $\sigma$-field $\mathscr{A}:=$ $\sigma\left(X_{k}, k \in Q\right)$, we now only need to prove that the r.v. $X_{(n, 0, \ldots, 0)}$ is independent of $\mathscr{A}$.

Let $A$ be an arbitrary atom of the (purely atomic) $\sigma$-field $\mathscr{A}$. To prove that $X_{(n, 0, \ldots, 0)}$ is independent of $\mathscr{A}$, it suffices to prove that $P\left(X_{(n, 0, \ldots, 0)}=\right.$ $y \mid A)=\frac{1}{2}$ for $y=-1,1$. It will be enough to go through the argument for $y=1$.

The event $A$ has the form $A:=\left\{X_{k}=z_{k} \forall k \in Q\right\}$, where $z_{k} \in\{-1,1\}$ for each $k$. By (2.1) and (2.2),

$$
X_{(n, 0, \ldots, 0)}=X_{j} \cdot X_{h} \cdot \prod\left[X_{k}: k \in T_{n}-\{h\}\right],
$$

where $j \in \mathbf{Z}^{d}$ is such that $m:=\|j\|<n$. Using (2.4), one has that $X_{h}$ is independent of $\mathscr{A}$. Defining the number $z:=z_{j} \cdot \prod\left[z_{k}: k \in T_{n}-\{h\}\right]$, we have that $z \in\{-1,1\}$, and hence

$$
P\left(X_{(n, 0, \ldots, 0)}=1 \mid A\right)=P\left(z \cdot X_{h}=1 \mid A\right)=P\left(X_{h}=z \mid A\right)=\frac{1}{2} .
$$

This completes the proof of Lemma 3.

Now suppose $i \in\{1, \ldots, d\}$ and $m$ is an integer. To prove that the random field $X$ is "lattice-halfspace one-dependent", it suffices to show that the $\sigma$-fields $\mathscr{G}:=\sigma\left(X_{k}, k \in G_{i, m-1}\right)$ and $\mathscr{H}:=\sigma\left(X_{k}, k \in H_{i, m+1}\right)$ are independent.

Define the set $V:=\mathbf{Z} \times \cdots \times \mathbf{Z} \times\{m\} \times \mathbf{Z} \times \cdots \times \mathbf{Z}$, where the set $\{m\}$ is in the $i$ th position. Then the sets $G_{i, m-1}, V, H_{i, m+1}$ partition $\mathbf{Z}^{d}$.

If $m=0$, then by an induction argument using Lemma 3 the r.v.'s $X_{k}$, $k \in \mathbf{Z}^{d}-V$, are independent, and it follows that the $\sigma$-fields $\mathscr{G}$ and $\mathscr{H}$ are independent.

Suppose $m \geq 1$. Then $V$ intersects each of the sets $S_{m}, S_{m+1}, S_{m+2}, \ldots$ but not $S_{0} \cup \cdots \cup S_{m-1}$. By an induction argument using Lemma 3, the $\sigma$-fields $\sigma\left(X_{k}\right), k \in\left(S_{m} \cup S_{m+1} \cup S_{m+2} \cup \cdots\right)-V$, and $\sigma\left(X_{k}, k \in S_{0} \cup \cdots \cup S_{m-1}\right)$ are independent. Since $S_{0} \cup \cdots \cup S_{m-1} \subset G_{i, m-1}$, it follows that $\mathscr{G}$ and $\mathscr{H}$ are independent.

If $m \leq-1$, then $\mathscr{G}$ and $\mathscr{H}$ are independent by a similar argument. Thus $X$ is "lattice-halfspace one-dependent", and the proof of Proposition 1 is complete.

\section{Proof of Theorem 1}

Olshen [14, p. 156, lines 4-12] employed a certain trick in order to "convert" a nonstationary random sequence with certain properties (trivial "past" and "future" tail $\sigma$-fields, a nontrivial "double" tail) into a stationary sequence with 
those properties. Olshen's trick was used later on in [1, §3], and it will be used again here.

For each $j \in \mathbf{Z}^{d}$, let $X^{(j)}:=\left(X_{k}^{(j)}, k \in \mathbf{Z}^{d}\right)$ be a random field with all of the properties specified in Proposition 1. Assume further that these random fields $X^{(j)}, j \in \mathbf{Z}^{d}$, are independent of each other and have the same distribution.

Let $\theta: \mathbf{Z}^{d} \rightarrow\left\{1 / 3,1 / 3^{2}, 1 / 3^{3}, \ldots\right\}$ be a one-to-one correspondence.

Define the random field $X:=\left(X_{k}, k \in \mathbf{Z}^{d}\right)$ as

$$
X_{k}:=\sum\left[\theta(j) \cdot X_{j}^{(k-j)}: j \in \mathbf{Z}^{d}\right] \quad \forall k \in \mathbf{Z}^{d} .
$$

Note that the sum converges absolutely. Also note that

$$
\sigma\left(X_{k}\right)=\sigma\left(X_{j}^{(k-j)}, j \in \mathbf{Z}^{d}\right) \quad \forall k \in \mathbf{Z}^{d} .
$$

It is clear that the $X_{k}$ 's are nondegenerate (in fact nonatomic) r.v.'s. The proof that the random field $X$ is strictly stationary can be spelled out somewhat informally as follows: The random field $Y:=\left(X^{(j)}, j \in \mathbf{Z}^{d}\right)$ of random fields is trivially strictly stationary. Suppose we define a function $f(Y):=$ $\sum\left[\theta(j) \cdot X_{j}^{(-j)}: j \in \mathbf{Z}^{d}\right]$. Then for each $k \in \mathbf{Z}^{d}$, one has $X_{k}=f\left(X^{(k+j)}\right.$, $\left.j \in \mathbf{Z}^{d}\right)$, simply a "shift" of $f(Y)$ by the vector $k$. Hence the random field $X$ is strictly stationary.

Next let us prove that $X$ is "lattice-halfspace one-dependent". Suppose $i \in$ $\{1, \ldots, d\}$ and $m$ is an integer. It suffices to prove that the $\sigma$-fields $\mathscr{G}:=$ $\sigma\left(X_{k}, k \in G_{i, m}\right)$ and $\mathscr{H}:=\sigma\left(X_{k}, k \in H_{i, m+2}\right)$ are independent. Let the $i$ th coordinate of any $h \in \mathbf{Z}^{d}$ be denoted $h_{i}$. Now by (3.2)

$$
\begin{aligned}
\mathscr{G} & =\sigma\left(X_{j}^{(h)},(h, j) \in \mathbf{Z}^{d} \times \mathbf{Z}^{d} \text { such that } h+j \in G_{i, m}\right) \\
& =\bigvee_{h \in \mathbf{Z}^{d}}\left[\sigma\left(X_{j}^{(h)}, j \in \mathbf{Z}^{d} \text { such that } j_{i} \leq m-h_{i}\right)\right] .
\end{aligned}
$$

Similarly

$$
\mathscr{H}=\bigvee_{h \in \mathbf{Z}^{d}}\left[\sigma\left(X_{j}^{(h)}, j \in \mathbf{Z}^{d} \text { such that } j_{i} \geq m+2-h_{i}\right)\right] .
$$

Now for each fixed $h \in \mathbf{Z}^{d}$ the two $\sigma$-fields in the brackets are independent. It follows that $\mathscr{G}$ and $\mathscr{H}$ are independent. Thus $X$ is "lattice-halfspace onedependent".

All that remains now is to show that the random field $X$ is "tail deterministic". Suppose $K \in \mathbf{Z}^{d}$ and $B$ is an arbitrary finite subset of $\mathbf{Z}^{d}$. It suffices to show that $X_{K}$ is measurable with respect to $\sigma\left(X_{k}, k \notin B\right)$.

By (3.2),

$$
\begin{aligned}
\sigma\left(X_{k}, k \notin B\right) & =\sigma\left(X_{j}^{(h)},(h, j) \in \mathbf{Z}^{d} \times \mathbf{Z}^{d} \text { such that } h+j \notin B\right) \\
& =\bigvee_{h \in \mathbf{Z}^{d}}\left[\sigma\left(X_{j}^{(h)}, j \in \mathbf{Z}^{d} \text { such that } j \notin B-h\right)\right],
\end{aligned}
$$

where $B-h=\{k-h: k \in B\}$, a finite set. For each $h \in \mathbf{Z}^{d}$, the r.v. $X_{K-h}^{(h)}$ is measurable with respect to the $\sigma$-field in the brackets. Since

$$
\sigma\left(X_{K}\right)=\sigma\left(X_{K-h}^{(h)}, h \in \mathbf{Z}^{d}\right)
$$

by (3.2), it follows that $X_{K}$ is measurable with respect to $\sigma\left(X_{k}, k \notin B\right)$. Thus $X$ is "tail deterministic". This completes the proof. 


\section{REFERENCES}

1. R. C. Bradley, A bilaterally deterministic $\rho$-mixing stationary random sequence, Trans. Amer. Math. Soc. 294 (1986), 233-241.

2. $\ldots$ A caution on mixing conditions for random fields, Statist. Probab. Lett. 8 (1989), 489-491.

3. $ـ$ On the spectral density and asymptotic normality of weakly dependent random fields, J. Theoret. Probab. 5 (1992), 355-373.

4. __ Equivalent mixing conditions for random fields, Ann. Probab. (in press).

5. A. V. Bulinskii, On various conditions of mixing and asymptotic normality of random fields, Soviet Math. Dokl. 37 (1988), 443-448.

6. __ Limit theorems under weak dependence conditions, Moscow Univ. Press, Moscow, 1989. (Russian)

7. R. Burton, M. Denker, and M. Smorodinsky, Finite state bilaterally deterministic strongly mixing processes, Israel J. Math. (submitted).

8. R. L. Dobrushin, The description of a random field by means of conditional probabilities and conditions of its regularity, Theory Probab. Appl. 13 (1968), 197-224.

9. V. F. Gaposhkin, Moment bounds for integrals of $\rho$-mixing fields, Theory Probab. Appl. 36 (1991), 249-260.

10. C. M. Goldie and P. Greenwood, Variance of set-indexed sums of mixing random variables and weak convergence of set-indexed processes, Ann. Probab. 14 (1986), 817-839.

11. V. V. Gorodetskii, The central limit theorem and an invariance principle for weakly dependent random fields, Soviet Math. Dokl. 29 (1984), 529-532.

12. V. M. Gurevic, On one-sided and two-sided regularity of stationary random processes, Soviet Math. Dokl. 14 (1973), 804-808.

13. C. C. Neaderhouser, An almost sure invariance principle for partial sums associated with a random field, Stochastic Process. Appl. 11 (1981), 1-10.

14. R. A. Olshen, The coincidence of measure algebras under an exchangable probability, $Z$. Wahrsch. Verw. Gebiete 18 (1971), 153-158.

15. D. S. Ornstein and B. Weiss, Every transformation is bilaterally deterministic, Israel J. Math. 21 (1975), 154-158.

16. M. Rosenblatt, Stationary sequences and random fields, Birkhäuser, Boston, MA, 1985.

17. L. T. Tran, Kernel density estimation on random fields, J. Multivariate Anal. 34 (1990), 37-53.

18. I. G. Zhurbenko, On mixing conditions for random processes with values in a Hilbert space, Soviet Math. Dokl. 30 (1984), 465-467.

19. _ The spectral analysis of time series, North-Holland, Amsterdam, 1986.

Department of Mathematics, Indiana University, Bloomington, Indiana 47405

E-mail address: bradleyr@ucs.indiana.edu 